\title{
A consistent view on star-forming galaxies at high redshift from multi-wavelength observations and SED modeling
}

\author{
Daniel Schaerer ${ }^{1,2}$, Stephane de Barros ${ }^{3}$ and Frederic Boone ${ }^{2}$ \\ ${ }^{1}$ Observatoire de Genève, Université de Genève, 51 Ch. des Maillettes, 1290 Versoix, \\ Switzerland email: daniel.schaerer@unige.ch \\ ${ }^{2}$ CNRS, IRAP, 14 Avenue E. Belin, 31400 Toulouse, France \\ ${ }^{3}$ INAF-Osservatorio Astronomico di Bologna, via Ranzani 1, 40127, Bologna, Italy
}

\begin{abstract}
We stress the importance of consistent SED analysis for distant star-forming galaxies (SFGs). We then summarise recent results from such an analysis concerning their basic physical properties, such as the determination of star formation rate (SFR), stellar mass, specific star SFR, UV attenuation, and how this affects our knowledge of star formation properties at high-z.
\end{abstract}

Keywords. Galaxies: starburst, Galaxies: high-redshift

\section{Introduction and need for consistent analysis}

Impressive progress has been achieved in recent years on observations of distant starforming galaxies (SFGs, hereafter), which have been identified in a variety of surveys, in particular with the HST. Although large numbers of SFGs are now known, many both basic and important questions remain open or at least uncertain for galaxies at $z \gtrsim 3$. For example, fundamental properties such as star formation rates (SFR), stellar mass, and probably even more so extinction, age, and metallicity of most high-z SFGs are still quite poorly known, and subject to various hypothesis (e.g. Conroy (2013), de Barros et al. (2014) hereafter DB14).

Related questions of interest and importance are the UV attenuation of the Lyman break, i.e. UV-selected galaxies, and more generally the amount of dust-obscured star formation at $z>3$. Indeed, above this redshift, no or few direct measurements of reprocessed emission from dust in the IR are available, and a measure of the average UV attenuation obtained from combining Herschel and other multi-wavelength data Burgarella et al. (2013) is limited to $z \lesssim 3.5$.

Also, the nature and physics of star formation at high redshift remains to be better understood and compared to lower redshift. For example, while star formation proceeds on a fairly "universal" timescale of $\sim 1$ Gyr at low $z$, it remains open how universal this property really is, i.e. what the SF timescale is at high redshift, and whether it is redshift dependent. For example, Salmi et al. (2012) and many others, find a small dispersion in the SFR- $M_{\star}$ relation, arguing for a steady, environment-independent mode of star formation, which may also extend to higher redshift (Speagle et al. (2014)). However, first but still sparse measurements of the molecular gas depletion timescale at different redshifts show a tentative decline of this timescale with increasing redshift (Saintonge et al. (2013), Dessauges-Zavadsky et al. (2015)). Also, simulations including different types of feedback show short term variations of the SFR at high redshift (e.g. Wyithe \& Loeb (2011), Hopkins et al. (2014)). Finally, as we have argued earlier and will discuss 
below, the dispersion in the SFR- $M_{\star}$ relation strongly depends on assumptions regarding the star formation histories and may indeed be quite large at high redshift.

If star formation is not steady in the early Universe, this has many important consequences. For example the commonly used SFR indicators, such as SFR(UV) or SFR(IR), are then not valid anymore, i.e. SFR-mass relations and their scatter may be different. This clearly calls for a consistent analysis of the observational data to determine the physical parameters of high redshift SFGs.

\section{Main implications from consistent SED modeling of high-z LBGs}

Including nebular emission and exploring a wide range of star formation histories (SFHs), we have examined in DB14 in detail how the physical parameters are affected by lines and assumptions on the SFH. We now briefly summarize the various main implications obtained from this approach which makes consistent use of broad-band photometry available for typical deep galaxy surveys covering the optical to near-IR domain.

SFR and mass: Figure 1 shows how different model assumptions affect stellar mass, SFR primarily, and hence the inferred scatter in the SFR-mass diagram for a sample of $z \sim 4$ LBGs. The upper panels shows, for reference, the results obtained with "standard" SED fits assuming constant SFR and an age prior of $\geqslant 50$ Myr. The other panels show the results for variable SFHs, i.e. exponentially declining or rising histories. Clearly the resulting scatter is larger than obtained for "standard" assumptions. As shown in DB14, the variable SFHs are overall favored, especially to reproduce the observed distribution of emission line $(\mathrm{H} \alpha)$ strengths probed by the IRAC photometry for these galaxies. New, additional constraints - most importantly direct measurements of the rest-frame optical emission lines - are needed to distinguish more firmly different cases and to determine the true scatter in SFR- $M_{\star}$.

The specific SFR: A natural outcome of SED fits applying the same methodology, assumptions and code at different redshifts is the finding of a rise of the average specific star formation rate, sSFR, with redshift, as illustrated in Fig. 1 (right) taken from DB14 and first shown in de Barros et al. (2011). Again, the exact increase of $\operatorname{sSFR}(\mathrm{z})$ and the predicted scatter depend on the assumed star formation history and on possible age priors (as for the SFR-mass relation). Similar results are found by other groups/methods (e.g. Stark et al. (2013)) and the behavior of $\operatorname{sSFR}(\mathrm{z})$ is qualitatively in agreement with galaxy evolution models.

UV attenuation: Another interesting result obtained consistently from the same SED analysis concerns the UV attenuation of LBGs, which is found to be higher than what would be inferred from the commonly-used UV slope measurement (cf. Bouwens et al. (2012)), which relies on the classical "Meurer relation" between the UV slope and attenuation, which in turn assumes constant SFR over durations longer than $\gtrsim 300 \mathrm{Myr}$ and solar metallicity. However, these assumptions are probably not fulfilled in typical high-z SFGs, whose stellar populations will mostly have sub-solar metallicities and be of younger age. Hence the intrinsic UV slope will be bluer, which explains the higher UV attenuation (Castellano et al. (2014), DB14). Both studies have also proposed a revised "Meurer law" which should be more appropriate for high-z SFGs. The resulting increase of the UV attenuation is typically by a factor $\sim 2$ at redshift $z \sim 3-6$ and lower at higher $z$, as illustrated in Fig. 2, which will be further discussed below (Sect. 3).

All major physical parameters derived from SED fits depend - to different extent - on assumptions made on star formation histories, possible age priors, and on the treatment of nebular emission. Furthermore there are degeneracies between different parameters. For these reasons it is important to examine consistently all the physical parameters and 

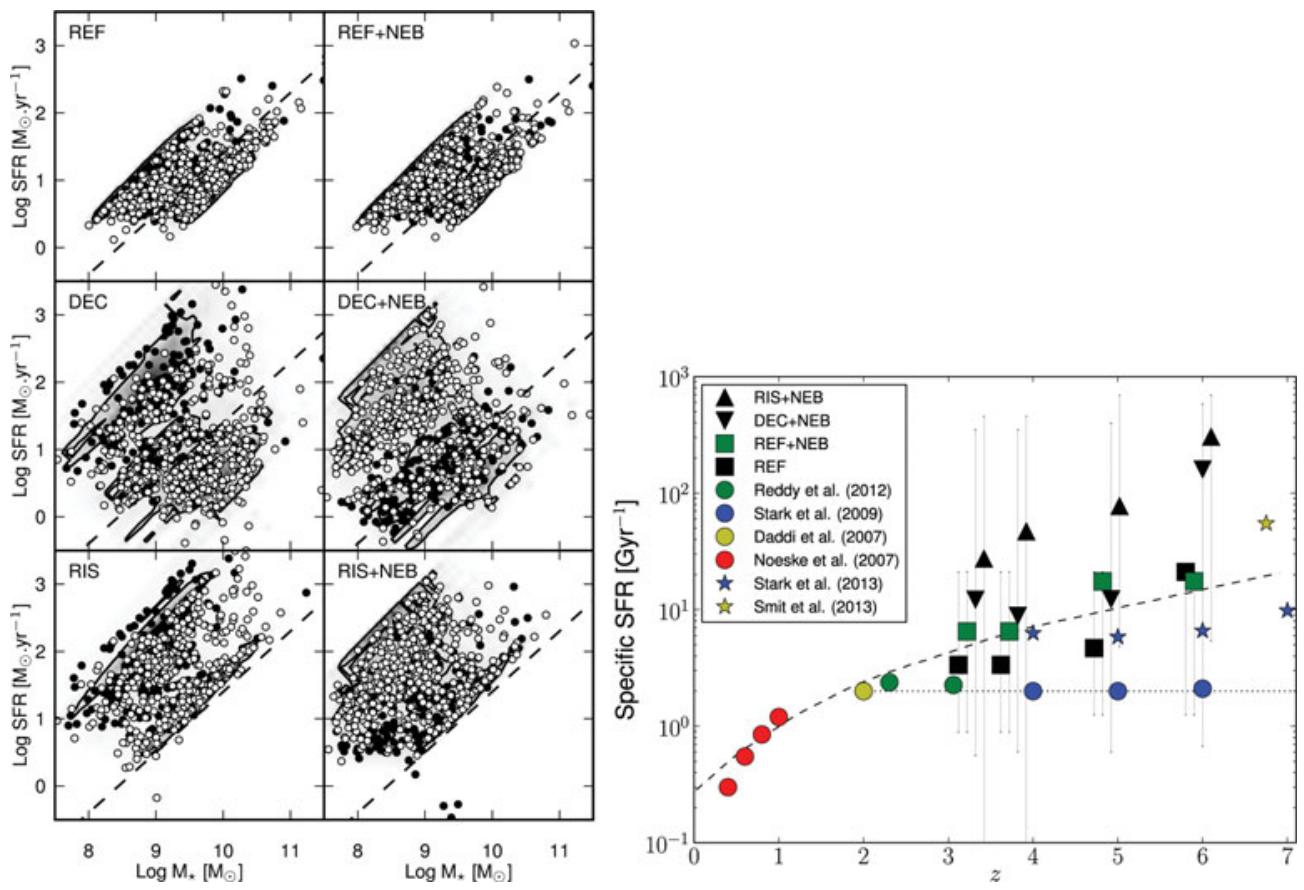

Figure 1. Left: SFR - stellar mass relations of $z \sim 4$ LBGs obtained from SED fits for different assumptions on the SFHs (top-to-bottom) and with/out nebular emission (right/left). Right: Evolution of the sSFR of LBGs with redshift derived for different model assumptions. Figs. taken from DB14; see there fore more details.

their uncertainties. To summarize, here is a list of physical quantities and some recent references discussing them for high-z star-forming galaxies:

- galaxy ages (cf. Schaerer \& de Barros (2009), Schaerer \& de Barros (2010), DB14)

- stellar masses (see DB14, Duncan et al. (2014), Grazian et al. (2015))

- SFR and scatter in SFR-mass relation (see above)

- sSFR evolution with redshift (see above)

- higher dust attenuation (see above)

- variable SFHs (see DB14, also Schaerer et al. (2013), Sklias et al. (2014))

\section{New observational constraints and future improvements}

Clearly, new data is required to significantly improve the current situation, i.e. to reduce existing degeneracies, and to provide direct measures of the instantaneous SFR (to compare with SFR(UV) measuring longer timescales) and the UV attenuation. Dust continuum data from ALMA for the latter, and rest-frame optical emission line measures (H $\alpha$ in particular) with the JWST for the former, are now or will soon be possible.

Figure 2 shows a comparison of the UV attenuation from recent ALMA and IRAM measurements of $z \gtrsim 5$ star-forming galaxies with our predictions and other data. The typical UV attenuation obtained from our SED fits for LBGs is between $\sim 1.1$ and $0.5 \mathrm{mag}$ for $z \sim 3.5-7$ (cf. Schaerer \& de Barros, in prep.). Although several of Capak's objects show a fairly low attenuation, two have $A_{\mathrm{UV}} \approx 1$.4. If confirmed Watson's object shows also a high UV attenuation. For now, given the small samples available and their very heterogeneous selection, it seems difficult to draw firm conclusions from this comparison with our models. In the near future this situation should rapidly change, providing thus 


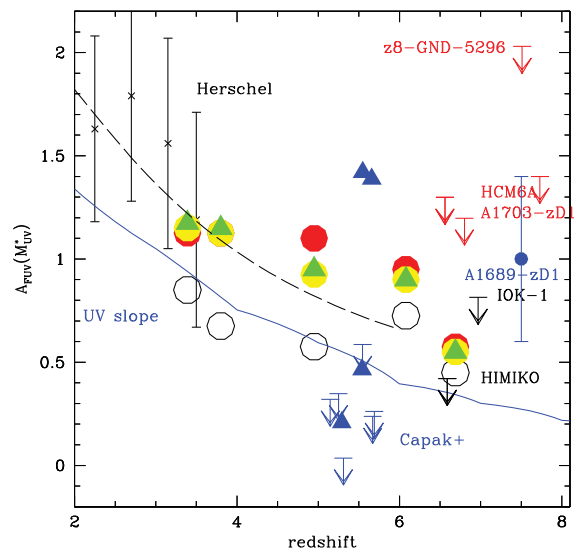

Figure 2. UV attenuation as a function of redshift for different SFGs: mean attenuation from IR/UV measurements with Herschel (Burgarella et al. (2013)) at $z \lesssim 3.5$ (black crosses with error bars and dashed line showing extrapolation to higher $z$ ); attenuation of LBGs at the characteristic UV magnitude $M_{\mathrm{UV}}^{\star}$ derived from the UV slope (blue solid line); mean attenuation at $M_{\mathrm{UV}}^{\star}$ of the LBG sample from DB14 derived from our SED fits (red/yellow circles and green triangles for different SFHs). The blue symbols show results from recent ALMA measurements of SFGs at $z \sim 5$ by Capak et al. (2015) and Watson et al. (2015) at $z=7.5$; other upper limits from ALMA and IRAM at $z \geqslant 6.5$ taken from Schaerer et al. (2015) are also shown.

important new insight of dust obscured SF and reducing significantly degeneracies in SED modeling (cf. Schaerer \& de Barros, in prep.).

\section{References}

Bouwens, R. J., Illingworth, G. D., Oesch, P. A., et al. 2012, ApJ, 754, 83

Burgarella, D., Buat, V., Gruppioni, C., et al. 2013, A\&A, 554, A70

Capak, P. L., Carilli, C., Jones, G., et al. 2015, Nature, 522, 455

Castellano, M., Sommariva, V., Fontana, A., et al. 2014, A\&A, 566, A19

Conroy, C. 2013, ARAA, 51, 393

de Barros, S., Schaerer, D., \& Stark, D. P. 2011, in SF2A-2011: Proceedings of the Annual meeting of the French Society of Astronomy and Astrophysics, ed. G. Alecian, K. Belkacem, R. Samadi, \& D. Valls-Gabaud, 95-99

de Barros, S., Schaerer, D., \& Stark, D. P. 2014, A\&A, 563, A81

Dessauges-Zavadsky, M., Zamojski, M., Schaerer, D., et al. 2015, A\&A, 577, A50

Duncan, K., Conselice, C. J., Mortlock, A., et al. 2014, MNRAS, 444, 2960

Grazian, A., Fontana, A., Santini, P., et al. 2015, A\&A, 575, A96

Hopkins, P. F., Kereš, D., Oñorbe, J., et al. 2014, MNRAS, 445, 581

Saintonge, A., Lutz, D., Genzel, R., et al. 2013, ApJ, 778, 2

Salmi, F., Daddi, E., Elbaz, D., et al. 2012, ApJL, 754, L14

Schaerer, D., Boone, F., Zamojski, M., et al. 2015, A\&A, 574, A19

Schaerer, D. \& de Barros, S. 2009, A\&A, 502, 423

Schaerer, D. \& de Barros, S. 2010, A\&A, 515, A73+

Schaerer, D., de Barros, S., \& Sklias, P. 2013, A\&A, 549, A4

Sklias, P., Zamojski, M., Schaerer, D., et al. 2014, A\&A, 561, A149

Speagle, J. S., Steinhardt, C. L., Capak, P. L., \& Silverman, J. D. 2014, ApJS, 214, 15

Stark, D. P., Schenker, M. A., Ellis, R., et al. 2013, ApJ, 763, 129

Watson, D., Christensen, L., Knudsen, K. K., et al. 2015, Nature, 519, 327

Wyithe, J. S. B. \& Loeb, A. 2011, MNRAS, 413, L38 